\title{
Vibrational Spectroscopy of Liquid Water by Monochromated Aloof EELS
}

Jacob R. Jokisaari, ${ }^{1 *}$ Jordan Hachtel, ${ }^{2}$ Xuan Hu, ${ }^{1}$ Arijita Mukherjee, ${ }^{1}$ Canhui Wang, ${ }^{1 * *}$ Andrea Konecna, ${ }^{3}$ Javier Aizpurua, ${ }^{3}$ Ondrej L.Krivanek, ${ }^{4}$ Juan-Carlos Idrobo, ${ }^{2}$ and Robert F. Klie ${ }^{1}$

1. University of Illinois at Chicago, Department of Physics, Chicago, IL 60607

2. Oak Ridge National Laboratory, Center for Nanophase Materials Science, Oak Ridge, TN 37831

3. Center for Material Physics, Donostia - San Sebastian Gipuzkoa 20018 SPAIN

4. Nion Company, 11511 NE $118^{\text {th }}$ St., Kirkland, WA 98034

*** now at: National Institute of Standards and Technology

The physics behind the behavior of water contains fundamental questions that are critical to many areas of science and engineering. Anomalous behavior in its phase diagram, questions in electrochemistry, and the effects of nanoscale confinement make up some of the more important topics of the moment. For example, in nanoscale confined volumes unique hydrogen bonding states have been identified[1], while in an aqueous medium, chemical reactions depend on the interaction of water with specific atomic scale surface sites[2]. If direct examination of the vibrational properties of water were possible with a spatial resolution of nanometers, systems such as adsorbed species, interfacial double-layers, or other ordered liquid structures could be investigated directly.

Historically, the most successful means of investigating bonding and local structure of water have been through vibrational spectroscopies, such as FTIR or Raman. Yet, the spatial resolution of these techniques is limited by light diffraction to several $\mu \mathrm{m}$, which is far larger than the scale on which many phenomena of interest occur. Electron microscopes, on the other hand, can readily achieve nanoscale spatial resolution, but their energy resolution has been insufficient to resolve vibrational spectra. With the development of monochromated electron microscopes, this limitation has been overcome. In our work reported here, we employed a new approach to directly study the vibrational spectra of water encapsulated in novel boron nitride liquid cells. A schematic and typical liquid cell is described in Figure 1. We demonstrate that the phonon spectra of bulk water as well as the effects of surfaces on the phonon spectra can be directly characterized at a spatial resolution of tens of nanometers. In addition, we demonstrate that our approach is sensitive to isotopes and we can easily distinguish between $\mathrm{H}_{2} \mathrm{O}$ and $\mathrm{D}_{2} \mathrm{O}$ liquid samples, shown in Figure 2. These results show good agreement with conventional spectroscopies, and with phenomenological modeling of the electron beam-specimen interaction.

Several innovations are key to these measurements. First, is that water readily decomposes under the electron beam, even at very low electron fluxes. This can be mitigated using the 'aloof' mode, where the beam is placed near $(10-50 \mathrm{~nm})$ but not on the sample while still getting an EELS signal via long range interactions[3]. A second issue involves the construction of the liquid cell. Graphene-based liquid cells have been utilized in many cases, but creates a problem for ultra low-loss EELS as graphene is a conductor and has a high background signal in the low loss region[4]. Here, this is mitigated by constructing liquid cells using insulating single-layer boron nitride as the window material. Finally, the phonon signal is weak and near the zero loss peak at energies of $0-3 \mathrm{eV}$, a cutting edge monochromated instrument is required to get the energy resolution and to suppress the tail of the zero loss peak. This combination has allowed us to directly detect the phonon stretching signals of liquid water with nanoscale resolution. We expect this technique to have broad applicability, with applications to medicine, biology and biological materials, catalysis, electrochemistry, and many other disciplines. 


\section{References:}

[1] Oleg Byl, et al, Jr., J. Am. Chem. Soc. 128, (2006) 12090-12097

[2] Somphonh P. Phivilay, Alexander A. Puretzky, Kazunari Domen, and Israel E. Wachs, ACS Catal., 3, (2013) 2920-2929

[3] Ondrej L. Krivanek, et al, Nature, 5 (2014), 209

[4] Canhui Wang, QiaoQiao, Tolou Shokuhfar, and Robert F. Klie, Adv. Mater., 26, (2014) 3410-3414

[5] Microscopy research performed as part of a user proposal at Oak Ridge National Laboratory's Center for Nanophase Materials Sciences (CNMS), which is a U.S. Department of Energy, Office of Science User Facility (J.A.H, J.C.I.).The acquisition of UIC JEOL JEMARM200CF was supported by an MRI-R ${ }^{2}$ grant from the National Science Foundation (DMR-0959470). The Gatan Quantum GIF acquisition at UIC is supported by an MRI grant from the National Science Foundation (DMR1626065). Theoretical simulations are supported by Spanish Ministry project FIS2016-80174-P. (A.K, J.A.)

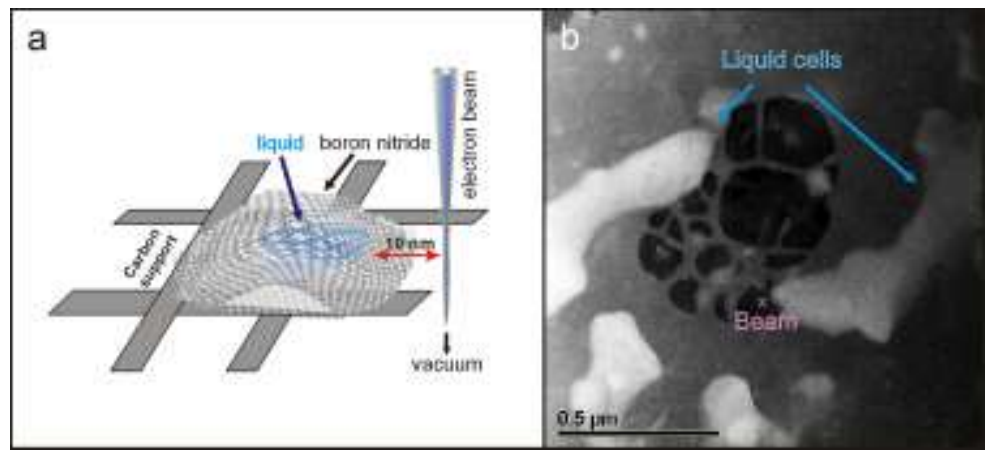

Figure 1. a) Schematic of a BN-encapsulated liquid cell. b) STEM image of representative liquid cells.

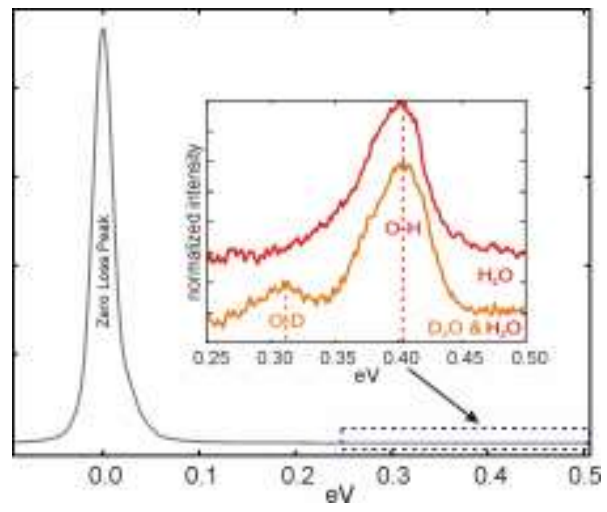

Figure 2. Typical monochromated EELS measurement showing the zero loss peak with a FWHM of $\sim 30 \mathrm{meV}$. The inset shows background subtracted EEL spectra of an $\mathrm{H}_{2} \mathrm{O}$ and a $\mathrm{D}_{2} \mathrm{O} / \mathrm{H}_{2} \mathrm{O}$ mixture which clearly show the signature $\mathrm{O}-\mathrm{H}$ stretching peak at $400 \mathrm{meV}$ and the D$\mathrm{O}$ peak at $310 \mathrm{meV}$. 